\section{International Scientific Journal Theoretical \& Applied Science}

\author{
p-ISSN: 2308-4944 (print) e-ISSN: 2409-0085 (online) \\ Year: $2016 \quad$ Issue: 5 Volume: 37 \\ Published: $30.05 .2016 \quad$ http://T-Science.org
}

SECTION 4. Computer science, computer engineering and automation.
Murat Sauranbayevich Tulenbayev Doctor of Technical Sciences, professor of Computer science, Dean of the Faculty Taraz State University named after M.Kh. Dulaty, Kazakhstan mtulenbaev@mail.ru

Svetlana Temirhanovna Beglerova Candidate of Science, Head of Sector Management of the Educational Process in the Distance Learning Department

(DLD)

Taraz State University named after M.Kh. Dulaty,

Kazakhstan

sbeglerova@mail.ru

Yelena Aleksandrovna Tuchina Master of Engineering science,

Software Engineer DLD

Taraz State University named after M.Kh. Dulaty,

Saule Tursinbayevna Aldashova Master of Engineering science,

Software Engineer DLD

Taraz State University named after M.Kh. Dulaty,

\title{
INTERACTIVE TRAINING COURSES FOR ENGINEERING EDUCATION BASED ON VIRTUAL LABORATORY COMPLEXES
}

Abstract: Considered are features of use and introduction of virtual laboratory complexes of distance technologies of engineering education within the project e-learning in Republic of Kazakhstan.

Key words: distance educational technologies, engineering education, virtual laboratory complexes.

Language: Russian

Citation: Tulenbayev MS, Beglerova ST, Tuchina YA, Aldashova ST (2016) INTERACTIVE TRAINING COURSES FOR ENGINEERING EDUCATION BASED ON VIRTUAL LABORATORY COMPLEXES. ISJ Theoretical \& Applied Science, 05 (37): 99-104.

Soi: http://s-o-i.org/1.1/TAS-05-37-19 Doi: crossef http://dx.doi.org/10.15863/TAS.2016.05.37.19

\section{ИНТЕРАКТИВЫЕ ОБУЧАЮЩИЕ КУРСЫ ИНЖЕНЕРНОГО ОБРАЗОВАНИЯ НА ОСНОВЕ ВИРТУАЛЬНЫХ ЛАБОРАТОРНЫХ КОМПЛЕКСОВ}

Аннотация: Рассматриваются особенности использования и внедрения виртуальных лабораторных комплексов дистанционных технологий инженерного образования в рамках проекта e-learning PK.

Ключевые слова: дистанционные образовательные технологии, инженерное образование, виртуальные лабораторные комплексы

В настоящее время существуют различные подходы к разработке интерактивных обучающих курсов. При этом можно выделить следующие основные направления в данной области: оформление учебных материалов в виде текстовых документов, слайдов компьютерных презентаций, гипертекстовых учебников, интерактивных анимационных элементов, справочных систем. Однако основными особенностями технических дисциплин в системах интерактивного и дистанционного обучения являются следующие: необходимость получения реальных практических умений и навыков работы с лабораторным оборудованием, а также, как правило, высокая стоимость такого оборудования, которая ограничивает возможности обеспечения каждого студента полным набором необходимых инструментальных средств.

Мультимедийные возможности современных программно-прикладных систем заключаются не только в расширении средств и методов представления информации, таких как графика, анимация, видео, звук, но и в иной 
форме организации данных, обусловленной возможностями программ. Так, в сфере профессионального образования помимо электронных учебников широкое признание получили виртуальные лабораторные работы.

При организации учебного процесса образовательными учреждениями РК в рамках проекта e-learning предполагается использовать технологии дистанционного обучения $[1,2]$ для подготовки специалистов по инженерным специальностям. На образовательном рынке РК возрождается интерес населения к инженернотехнологическим специальностям. Инженерное образование требует от студента не только усвоения определенного объема теоретических знаний, но и выработки навыков использования этих знаний на практике. Для приобретения таких навыков в учебных программах подготовки инженера много времени уделяется лабораторным и практическим работам, так как они способствуют укреплению теоретических знаний и повышению эффективности усвоения учебных материалов. Сегодня получение рентабельности производства подразумевает внедрение передовых технологий, стремительное развитие которых, в свою очередь, предполагает частую смену технологического оборудования на более совершенные. Поэтому ВУЗам в условиях жесткой конкуренции выпускаемых кадров необходимо обеспечить техническую лабораторную базу, отвечающую всем требованиям технологий сегодняшнего дня и оснащенную новейшими приборами и устройствами.

Одной из главных причин неразвитости рынка электронного обучения [3] для инженерного образования является техническая сложность освоения важнейших, ориентированных на практику, составляющих рабочих учебных планов. Возможность дистанционного обучения инженерным специальностям в ВУЗах в настоящее время может быть частично решена разработкой виртуальных и удаленных лабораторных практикумов. В качестве универсальной инструментальной среды могут быть использованы разработки лабораторий Таразского государственного университета имени М.Х.Дулати Информационно-обучающая система нашего Университета включает в себя программную и аппаратную платформы, что позволяет ее использовать в качестве универсальной среды для электронного обучения в инженерно-технологическом ВУЗе, включая виртуальный и удаленный лабораторный практикум. Эти технологии могут применяться не только для выполнения лабораторных работ и практических занятий, но и для курсового и дипломного проектирования, а также для подготовки магистерских и $\mathrm{PhD}$ диссертаций [4].

Виртуальная лабораторная работа заменяет (полностью или на определённых этапах) натуральный объект исследования, что позволяет гарантированно получить результаты опытов, избежать нанесения вреда живым организмам, сфокусировать внимание на ключевых сторонах исследуемого явления, сократить время проведения эксперимента. При проведении работы необходимо помнить, что виртуальная модель отображает реальные процессы и явления в более или менее упрощённом, схематичном виде, поэтому выяснение вопроса, что на самом деле подчёркнуто в модели, а что осталось за кадром, может быть одной из форм задания. Такого вида работы можно выполнять целиком в компьютерном варианте или сделать одним из этапов в более широкой работе, которая включает также работу с натуральными объектами и лабораторным оборудованием.

Виртуальный лабораторный практикум представляет собой интерактивный программный модуль, призванный реализовать переход от информационно-иллюстративной функции цифровых источников к функции инструментально-деятельностной и поисковой, как способствующей развитию критического мышления, выработке навыков и умений практического использования получаемой информации. [5] Примеры виртуальных лабораторных комплексов и практикумов есть как на дисках некоторых фирм-разработчиков программных продуктов для образовательных целей, так и в сети Интернет. В основу классификации лабораторных комплексов в системе отношений «преподаватель виртуальная лаборатория - студент» рационально положить характер модели, который во многом определяет подходы к использованию:

1. Качественная - явление или опыт, обычно сложные или невыполнимые в условиях учебного заведения, воспроизводится на экране при управлении пользователем;

2. Полуколичественная - в виртуальной лаборатории моделируется опыт, и реалистичное изменение отдельных характеристик (например, положение ползунка реостата в электрической цепи) вызывает изменения в работе установки, схемы, устройства;

3. Количественная (параметрическая) - в модели численно заданные параметры изменяют зависящие от них характеристики или моделируют явления.

Возможно создание и использование всех трех типов, но основной выбор за реалистичными полуколичественными лабораторными комплексами, обеспечивающие высокую эффективность их применения[6]. Так в ТарГУ 
используется лабораторный стенд «Микропроцессорные средства автоматизации и управления» ТОО "Chip Engineering LTD", который представляет собой реальное регулирующее управляющее оборудование (на основе программируемого логического контроллера SIMATIC S7-200, который запрограммирован как ПИД-регулятор) в сочетании с виртуальной моделью объекта (рисунок 1а). Виртуальная модель объекта регулирования реализована на основе MS Visual Basic.

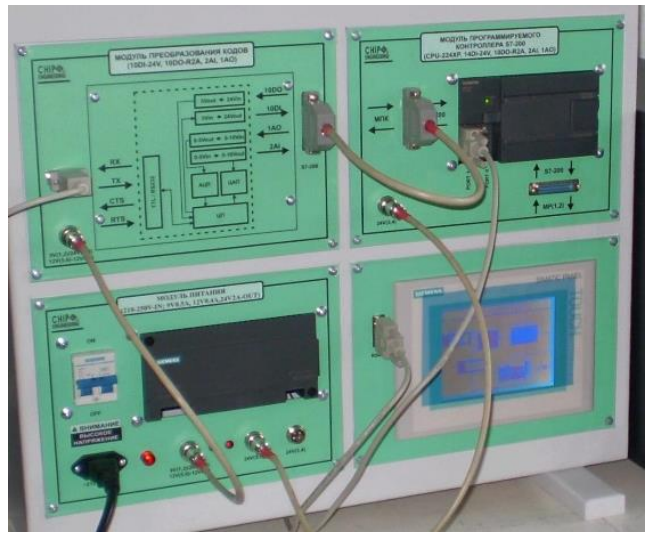

Другой типовой комплект учебного оборудования «Персональный компьютер» ПК-02 предназначен для функционирования, имитации и поиска неисправностей современного компьютера (рисунок 1б).

$$
\text { Следующий комплект учебного }
$$
оборудования «Сетевая безопасность» предназначен для: межсетевого экранирования с учетом состояния соединений; глубокого анализа протоколов прикладного уровня; трансляции сетевых адресов; IVPN; SSL VPN4 RIP, EIGRP, OSPF (рисунок 2a).

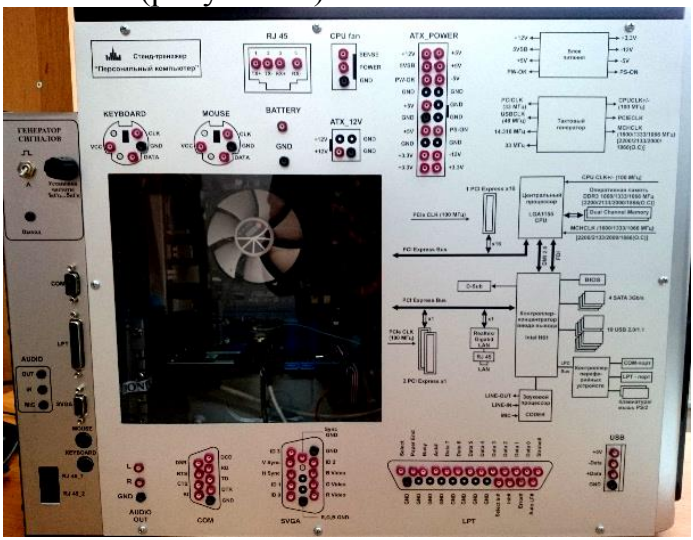

Рисунок 1 - Типовые комплекты учебного оборудования а) «Микропроцессорные средства автоматизации и управления», б) «Персональный компьютер» ПК-02.

Следующий типовой комплект учебного оборудования «Криптография» предназначен для изучения криптографических средств, применяемых при организации защиты информации (рисунок 2б): шифрование, хэширование, обмен ключами.

Проанализировав структуру значительного числа уже созданных виртуальных лабораторных работ по дисциплинам инженерного профиля, можно сделать заключение, что целями применения средств компьютерной графики в большинстве случае являются:

- приобретение студентами основных навыков восприятия графической информации, ее считывания и адаптации для принятия проектных решений;

- освоение студентами методов компьютерного моделирования отдельных деталей, узлов, машин и механизмов - от концептуального проектирования до сертификации готовой продукции;

- практическое применение студентами методов компьютерной инженерии, т.е. совокупности методов и средств практического решения инженерных задач, обычно выполняемых в реальных производственных условиях с помощью средств вычислительной техники.

Исходя из этого предлогаем программные и технические решения по разработке виртуальных лабораторий.

Electronics Workbench - предназначен для моделирования цифровых и аналоговых электронных схем. Electronics Workbench может проводить анализ схем на постоянном и переменном токах. Результаты этого анализа не отражаются на приборах, они используются для дальнейшего анализа схемы. B Electronics Workbench можно исследовать переходные процессы при воздействии на схемы входных сигналов различной формы. Программа также позволяет строить и производить анализ цифроаналоговых и цифровых схем большой степени сложности. Имеющиеся в программе библиотеки включают в себя большой набор широко распространенных электронных компонентов. (рисунок 3).

TRACE MODE - это программный комплекс, предназначенный для разработки и запуска в реальном времени распределенных автоматизированных систем управления технологическими процессами (АСУТП) (рисунок 4) и решения ряда задач управления предприятием (АСУП) [7].

ISPC Science and mechanics, 


\begin{tabular}{l|lrl|l|ll} 
& ISRA (India) & $=\mathbf{1 . 3 4 4}$ & SIS (USA) & $=\mathbf{0 . 9 1 2}$ & ICV (Poland) & $=\mathbf{6 . 6 3 0}$ \\
Impact Factor: & ISI (Dubai, UAE) $=\mathbf{0 . 8 2 9}$ & PUHU (Russia) $=\mathbf{0 . 2 3 4}$ & PIF (India) & $=\mathbf{1 . 9 4 0}$ \\
& GIF (Australia) & $\mathbf{0 . 5 6 4}$ & ESJI (KZ) & $=\mathbf{1 . 0 4 2}$ & IBI (India) & $\mathbf{4 . 2 6 0}$
\end{tabular}
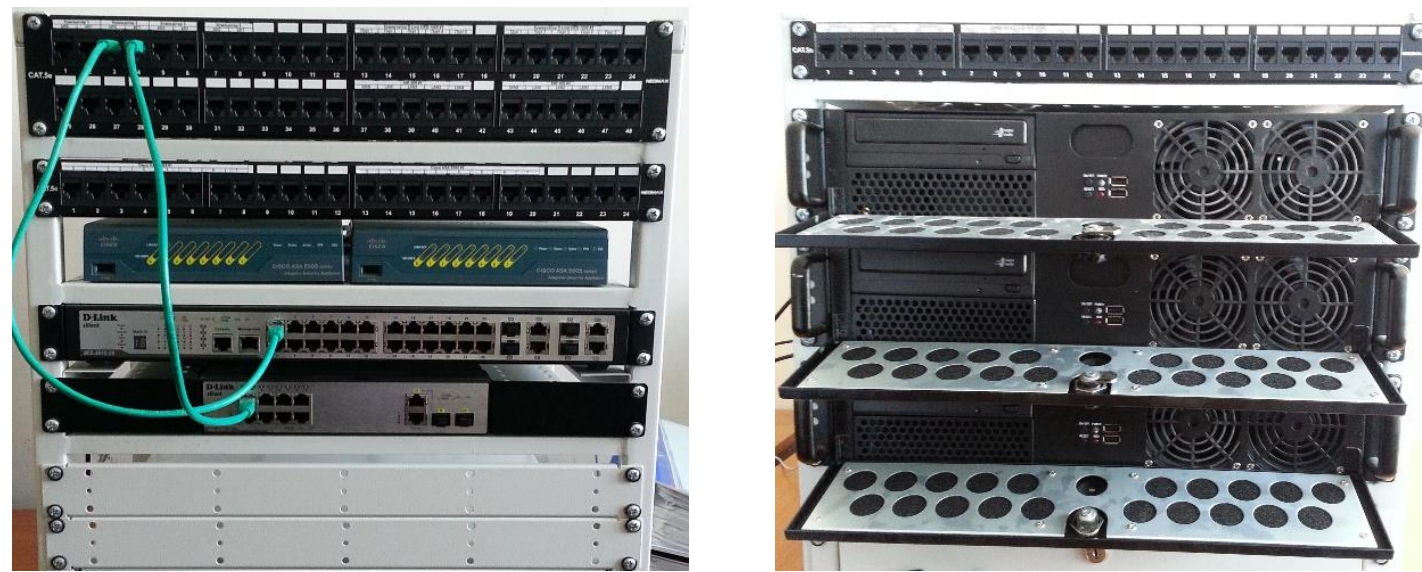

Рисунок 2 - Комплекты учебного оборудования: а) «Сетевая безопасность», б) «Криптография»

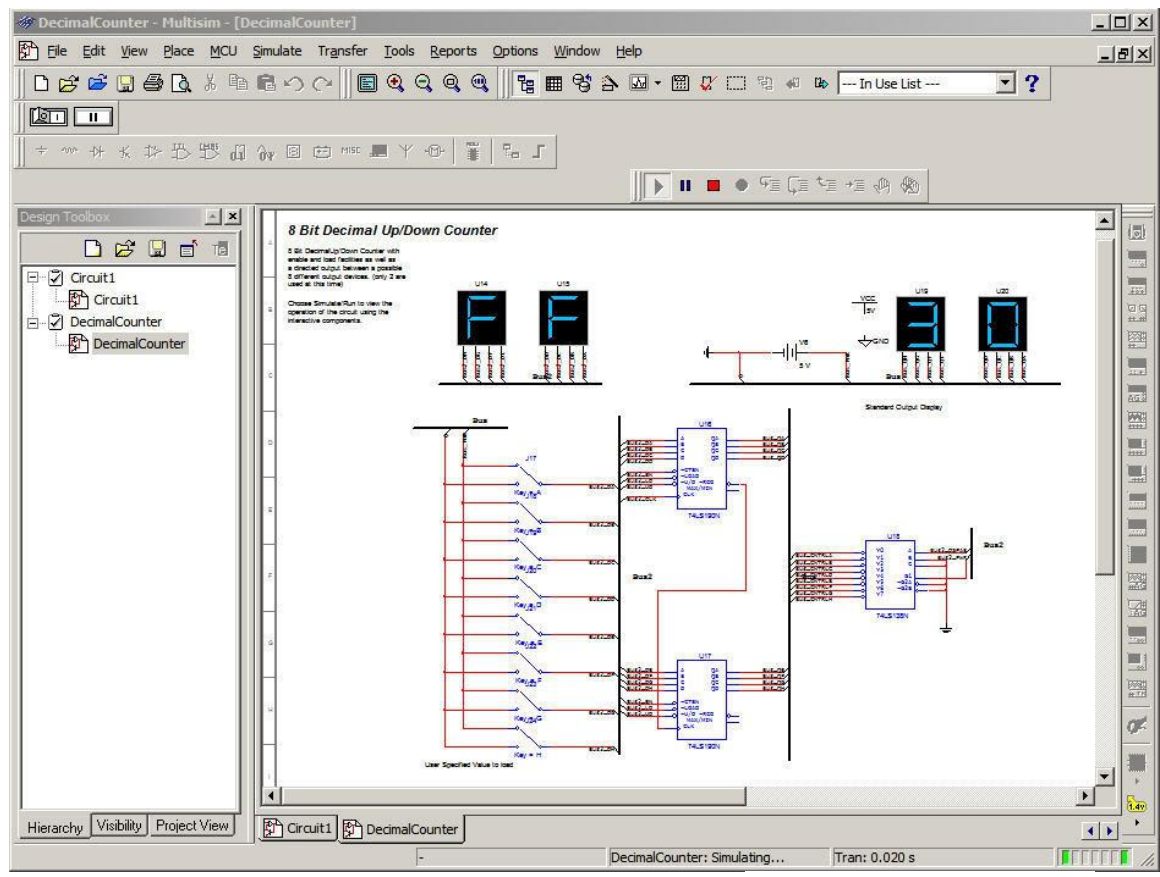

Рисунок 3 - Пример схемы построенной в Electronics Workbench

Интегрированная среда разработки проекта TRACE MODE - единая программная оболочка, содержащая все необходимые средства для разработки проекта.

Особенностью TRACE MODE является «технология единой линии программирования», то есть возможность разработки всех модулей АСУ при помощи одного инструмента. Технология единой линии программирования позволяет в рамках одного проекта создавать средства человеко-машинного интерфейса, системы учёта ресурсов, программировать промышленные контроллеры и разрабатывать web-интерфейс.
LabVIEW (англ. Laboratory Virtual Instrumentation Engineering Workbench) - это среда графического программирования, которую используют технические специалисты, инженеры, преподаватели и ученые по всему миру для быстрого создания комплексных приложений в задачах измерения, тестирования, управления, автоматизации научного эксперимента и образования (рисунок 5). Это среда разработки и платформа для выполнения программ, созданных на графическом языке программирования «G» фирмы National Instruments $[8,9]$. 


\begin{tabular}{l|lrl|l|ll} 
& ISRA (India) & $=\mathbf{1 . 3 4 4}$ & SIS (USA) & $=\mathbf{0 . 9 1 2}$ & ICV (Poland) & $=\mathbf{6 . 6 3 0}$ \\
Impact Factor: & ISI (Dubai, UAE) $=\mathbf{0 . 8 2 9}$ & PUHU (Russia) $=\mathbf{0 . 2 3 4}$ & PIF (India) & $=\mathbf{1 . 9 4 0}$ \\
& GIF (Australia) & $\mathbf{0 . 5 6 4}$ & ESJI (KZ) & $=\mathbf{1 . 0 4 2}$ & IBI (India) & $\mathbf{4 . 2 6 0}$
\end{tabular}

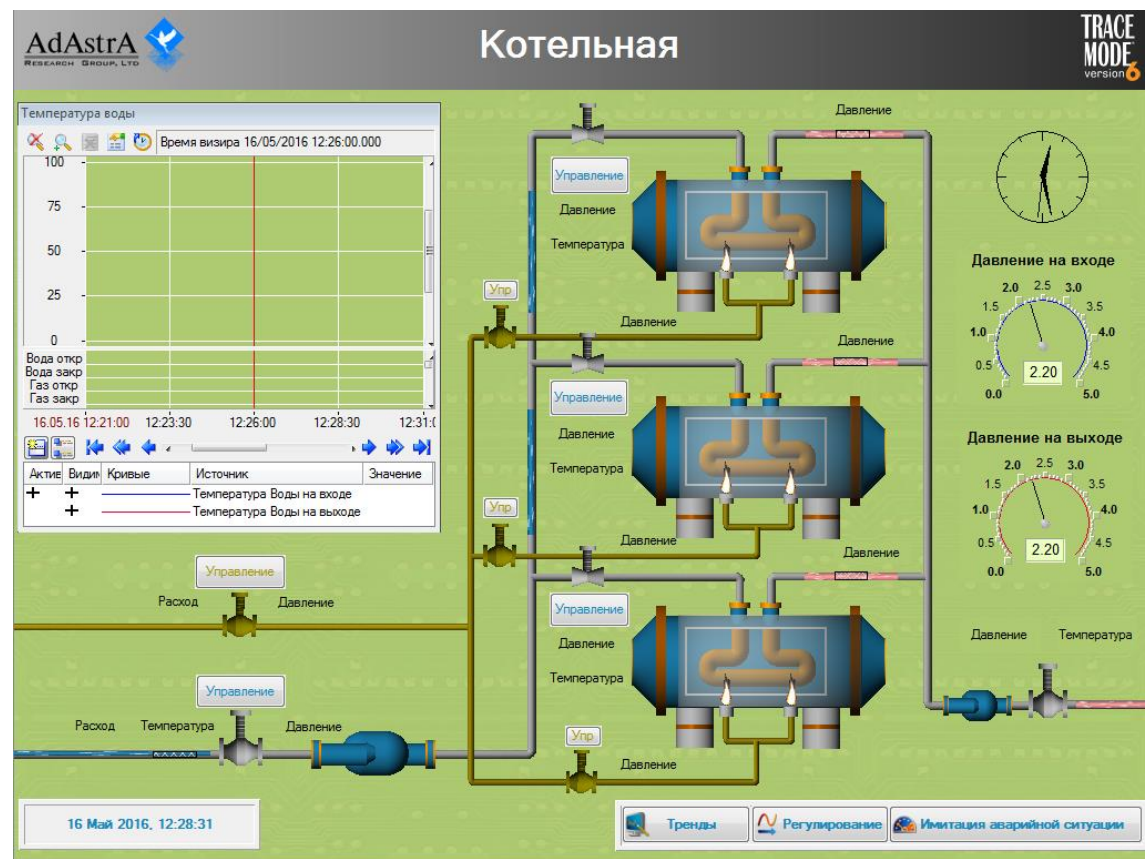

Рисунок 4 - Пример АСУTП в TRACE MODE

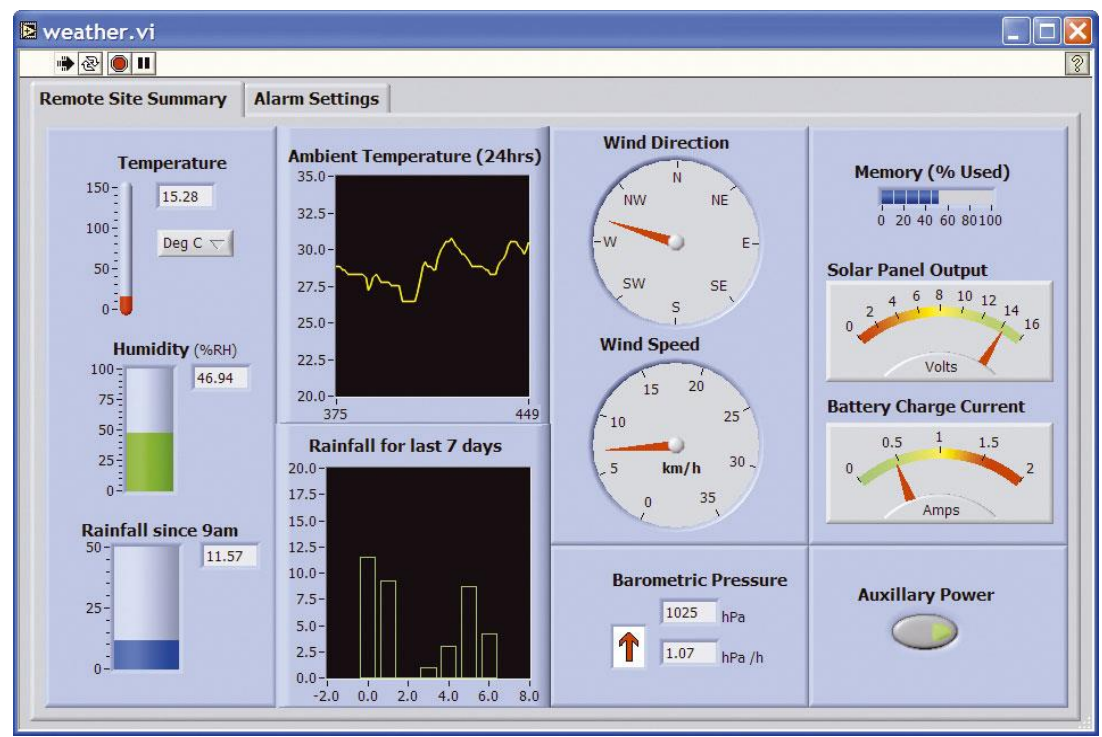

Рисунок 5 - Пример разработки приложения в LabVIEW

Отличительной особенностью используемой разработки является высокая реалистичность экспериментов в виртуальных лабораториях, точность воспроизведения физических законов мира и сущности опытов и явлений, а также уникально высокая интерактивность [10].

В отличие от реализованных виртуальных лабораторных работ, в которых отрабатываются не те умения и навыки, что в реальных работах, при создании реалистичных полуколичественных моделей упор сделан на формирование навыков экспериментальной работы, что актуально и целесообразно.

Исследование полуколичественной модели (c неявной математической основой) представляет собой нетривиальную задачу, в которую вовлекаются разнообразные умения: планировать эксперимент, выдвигать или выбирать наиболее разумные гипотезы о связи явлений, свойств, параметров, делать выводы на основе экспериментальных данных, формулировать задачи.

Особенно важным и целесообразным является умение указывать границы (область, условия) применимости научных моделей, включая изучение того, какие аспекты реального явления компьютерная модель воспроизводит удачно, а какие оказываются за гранью моделируемого.

ISPC Science and mechanics,

Lancaster, USA 


\begin{tabular}{l|lrl|l|ll} 
& ISRA (India) & $=\mathbf{1 . 3 4 4}$ & SIS (USA) & $=\mathbf{0 . 9 1 2}$ & ICV (Poland) & $=\mathbf{6 . 6 3 0}$ \\
Impact Factor: & ISI (Dubai, UAE) $=\mathbf{0 . 8 2 9}$ & PUHU (Russia) $=\mathbf{0 . 2 3 4}$ & PIF (India) & $=\mathbf{1 . 9 4 0}$ \\
& GIF (Australia) & $\mathbf{0 . 5 6 4}$ & ESJI (KZ) & $=\mathbf{1 . 0 4 2}$ & IBI (India) & $\mathbf{4 . 2 6 0}$
\end{tabular}

\section{References:}

1. Ibragimov IM (2007) Informacionnie tehnologii i sredstva distancionnogo obucheniya : ucheb. po-sobie dlya stud. VUZov [Tekst] / I.M. Ibragimov.- M.: Izdatelskii centr «Akademiya», 2007. -336 p.

2. Alekseev AN (2005) Distancionnoe obuchenie inzhenernim specialnostyam [Tekst] / A.N. Alekseev - Sumi: ITD «Universitetskaya kniga», 2005. -333 p.

3. Vul VA (2001) Elektronnie izdaniya: Uchebnik [Tekst] / V.A. Vul - SPb.:Izd-vo «Peterburgskii in-stitut pechati», 2001. $-308 \mathrm{p}$.

4. Tulenbaev MS, Temirgaliev TK, Dzhunisbekov MS (2011) Osobennosti ispolzovaniya virtualnih laboratornih kompleksov distancionnih obrazovatelnih tehnologii // Materiali mezhdunar. nauchno-prakt. konf. «Distancionnie tehnologii V obrazovanii 2011». - Karaganda, 2011. - pp. 146-148.

5. Dzhunisbekov MS, Temirgaliev TK (2012) Problemi i perspektivi razvitiya inzhenernogo obrazo-vaniya. // Uchebnoe posobie. Taraz:«Taraz universiteti», 2012. - 95p.
6. Tulenbaev MS, Tuchina EA, Aldashova ST (2015) Innovacionnie obrazovatelnie tehnologii SMART v VUZe // Materiali Resp. nauchnoprakt. konf. «Nauka i sovremennost - 2015», Mart. - Taraz, 2015. - T2. - pp.291-294

7. Morokina GS, Umbetov UU (2015) Uchebnoe posobie «Osnovi proektirovaniya priborov $\mathrm{i}$ informacionno-izmeritelnih sistem». - Taraz: Format-Print, 2015. - 168 p.

8. Trevis D, Kring D (2008) LabVIEW dlya vseh - M.: DMK Press, 2008 - 800 p.

9. Butirin PA, Vaskovskaya TA, Karataev VV, Materikin SV (2005) Avtomatizaciya fizicheskih issledovanii i eksperimenta: kompiuternie izmereniya $\mathrm{i}$ virtualnie pribori na osnove LabVIEW 7 (30 lekcii) / pod red. Butirina P.A. - M.: DMK Press, 2005. - 264 pp.

10. Sarsenbaeva GM (2010) Perspektivi razvitiya inzhenernogo obrazovaniya dlya industrialnoinnovacionnogo razvitiya Kazahstana // Kazahskii Nacionalnii Tehnicheskii Universitet imeni K.I.Satpaeva, Inzhener-noe Obrazovanie, 2010 g. - №6. - pp. 102-106. 